\title{
Performance of an independent planar virtual sound barrier at the opening of a rectangular enclosure
}

Jiancheng Tao ${ }^{a, b, *}$, Shuping Wang ${ }^{a}$, Xiaojun Qiu ${ }^{a}$, and Jie Pan ${ }^{b}$

${ }^{a}$ Key Laboratory of Modern Acoustics and Institute of Acoustics, Nanjing University, Nanjing, 210093, China

${ }^{b}$ School of Mechanical and Chemical Engineering, The University of Western Australia, WA 6009, Australia 


\section{ABSTRACT}

Planar virtual sound barrier systems have been used successfully to reduce noise radiation through an opening without affecting natural ventilation and lighting. However, the complexity of a fully coupled control system grows at the rate proportional to the square of the number of channels and this make the system implementation become impractical for enclosures with large openings. To reduce the system complexity, this paper proposes an independent planar virtual sound barrier, which is a multi-channel system consisting of many independent single channel active noise control systems. Each single channel system is "independent" in the sense that the control source output of the system is updated only with the signal from its own error sensor. Based on the analytical model of sound radiation through the opening of a rectangular enclosure, the transfer functions from both primary and control sources are calculated first. Then the noise reduction performance, the stability, and the convergence behavior of both fully coupled and independent planar virtual sound barrier systems are investigated. It is found that the independent system with no control output constraint becomes inherently unstable at some frequencies; however its stability can be improved by applying some control output constraint. Reducing the number of channels and the distance between secondary loudspeakers and error microphones can also increase system stability but at the cost of smaller noise reduction. When the system is inherently stable and there is no constraint on control output, the independent system can provide the same noise reduction as the fully coupled one but with faster convergence speed.

Keywords: virtual sound barrier, active noise control, open enclosure 


\section{Introduction}

A virtual sound barrier is an array of sound sources and microphones forming an acoustic barrier, which blocks direct propagation of sound while allowing air and light to pass through [1]. It is a multi-channel active noise control system and previous studies have shown that such a system can create a quiet zone inside a given space when the boundary is surrounded by error microphones [2-4]. Current virtual sound barrier systems are typically fully coupled adaptive systems, where the transfer functions from all secondary control sources to all error sensors are employed in the adaptive algorithm of the controller. The computation load of such a fully coupled controller grows at the rate proportional to the square of the number of channels and therefore decentralized systems have been proposed to reduce the system complexity [4]. Decentralized systems can reduce the computation complexity of the system by updating the outputs of some control sources based on the inputs of some error sensors but have weakness of stability and deteriorate performance. The "independent" system discussed in this paper is a special type of the decentralized systems, which only uses one collocated error sensor to update its corresponding control source.

A planar virtual sound barrier (PVSB) is a virtual sound barrier with planar senor and actuator arrays in parallel [5-6]. Because of its planar geometry, a PVSB system can be designed and applied conveniently at an opening of an enclosure to prevent sound radiation from or sound transmission into the enclosure through the opening with little influence on natural ventilation and lighting [7-9]. Four single channel active noise control systems (being called Active Acoustic Shielding (AAS) cells) were used to constitute an independent PVSB system at a $0.25 \mathrm{~m} \times 0.25 \mathrm{~m}$ window and the noise reduction in the receiving room was around 10 15 dB at frequencies between $500 \mathrm{~Hz}$ and $2 \mathrm{kHz}$ [7]. Sixteen single channel active noise control systems were installed at an open window to attenuate the noise radiated from outside to inside, and a noise reduction of more than $10 \mathrm{~dB}$ was achieved in a frequency range of 200 700 Hz [8]. A fifteen-channel fully coupled PVSB system was installed at a $5 \mathrm{~m}^{2}$ 
opening to investigate its noise reduction effect on transformer noise, and $16 \mathrm{~dB}$ and $7.7 \mathrm{~dB}$ noise reductions were achieved at $100 \mathrm{~Hz}$ and $200 \mathrm{~Hz}$ respectively in the far field [9]. These investigations showed that PVSB systems are effective for reducing noise radiation through an opening. However, the stability and the convergence behavior of PVSB systems have not been discussed.

Elliot et al. studied the convergence behavior of a multiple channel feedforward active control system in the frequency domain and proposed to describe this convergence in terms of the eigenvalues of the relevant Hessian matrix. In their study, both the sum of the mean-squared outputs of the error sensors and the sum of the mean-square inputs to the secondary sources were included in the cost function, and the coefficient of the latter term was defined as the weighting factor. It was pointed out that the weighting factor can change the convergence speed and provides a tradeoff between noise reduction performance and the output strength $[10,11]$.

Elliot and Boucher also analyzed the interaction between multiple feedforward active noise control systems in the frequency domain using the gradient descent algorithm and proposed a condition that is sufficient but not necessary for stability assessment based on the Gerschgorin circle theorem for the independently operating systems [12]. This condition is useful for a decoupled two-channel active noise control system in a free field, but the minimum interval between the error microphone and its corresponding secondary loudspeaker required for stability is much larger than the actual value when the number of channels of the control system increases. Although the derived condition is conservative, the eigenvalues analysis method can be adopted to assess the stability of independent PVSB systems.

Cordioli et al. showed that decentralizing an active control system for an electrical transformer in a free field can considerably reduce the steady state performance of the system via numerical simulations [13]. It was also found that decentralized systems can be stabilized by increasing the weighting factor, and there is an optimal weighting factor that results in the 
best noise reduction performance. However, there was no further analysis on the stability and convergence properties of the decentralized systems in their paper.

Yu et al. proposed a cluster configuration (actually a decentralized system), where the multichannel system is divided into several independent subsystems and each subsystem is fully coupled, to balance the complexity and instability for tonal noise control [14]. They found that the interval between subsystems should be larger than half the distance between the secondary loudspeaker and its corresponding error microphone in the subsystems for maintaining the stability where the two subsystems of two channels are placed "face to face" symmetrically in a free space. Unfortunately, this result cannot be applied to the independent PVSB system where the error microphone and the control loudspeaker in each independent channel are arranged facing towards the same direction.

The stability of decentralized feedback active control systems has also been investigated by some researchers [15-17]. Leboucher et al. analyzed the convergence of the adaptive process and the stability of the feedback loop of a decentralized adaptive feedback active control system, and deduced two stability conditions from the small gain theorem and the Nyquist criterion [15]. Grosdidier and Morari proposed a new interaction measure based on the structured singular value to predict the stability and the performance loss for decentralized feedback systems [16]. Zhang et al. studied the performance of decentralized multi-channel feedback analog control systems and obtained a sufficient stability condition in terms of the predefined maximum noise amplification and the geometrical configuration of the independent controllers [17].

Despite all the research mentioned in the preceding text, no research has been found on the performance especially the convergence rate of the feedforward independent PVSB system for controlling noise radiation through the opening of an enclosure. This will be investigated in this paper. First, the transfer functions are calculated based on an analytical radiation model of a monopole source inside a rectangular enclosure with one opening. Then 
the noise reduction performance, the stability resulting from the geometric arrangement of the error microphones and control loudspeakers, and the convergence of the adaptation process of the independent PVSB system is studied and compared to those of the fully coupled system. Finally, experiments are conducted to verify the conclusions.

\section{Theory}

\subsection{Sound radiation through the opening of a rectangular enclosure}

Figure 1 shows a simplified model for predicting the sound radiation through the opening of an enclosure, where a monopole located inside at $\mathbf{r}_{s}=\left(x_{s}, y_{s}, z_{s}\right)$ with a strength of $q_{0}$ is used to approximate the primary noise source. The enclosure dimensions are $L_{x}, L_{y}$, and $L_{z}$, and the opening surface is at $z=L_{z}$. A secondary loudspeaker array on the plane $z=L_{\mathrm{c}}$ and an error microphone array on the plane $z=L_{z}$ are used to constitute the PVSB system.

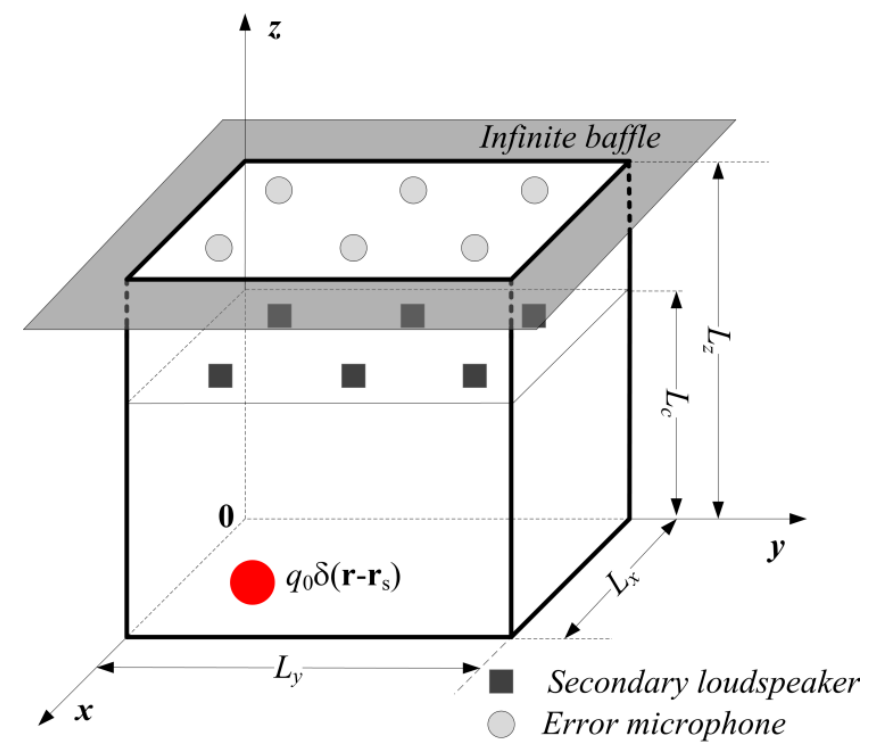

Fig. 1. A monopole sound source inside a rectangular enclosure with a baffled opening.

Assuming that the opening of the enclosure is embedded in an infinite baffle, the sound pressure amplitudes generated by the monopole source in frequency domain can be calculated with [18] 


$$
\begin{aligned}
& \quad p_{\text {in }}(x, y, z)=\sum_{n}\left(A_{n}^{\mathrm{i}} e^{-\mathrm{j} k_{n z} z}+A_{n}^{\mathrm{r}} e^{-\mathrm{j} k_{n z} z}\right) \phi_{n}(x, y)+\rho_{0} \omega q_{0} \sum_{n} \frac{\phi_{n}(x, y) \phi_{n}\left(x_{\mathrm{s}}, y_{\mathrm{s}}\right)}{2 S \Lambda_{n} k_{n z}} e^{-\mathrm{j} k_{n z}\left|z-z_{\mathrm{s}}\right|} \text { and } \\
& p_{\text {out }}(x, y, z)= \\
& \iint_{S_{0}}\left[-\mathrm{j} k_{n z}\left(-A_{n}^{\mathrm{i}} e^{-\mathrm{j} k_{n z} L_{z}}+A_{n}^{\mathrm{r}} e^{-\mathrm{j} k_{n z} L_{z}}\right) \phi_{n}\left(x_{0}, y_{0}\right)-\mathrm{j} \rho_{0} \omega q_{0} \frac{\phi_{n}\left(x_{0}, y_{0}\right) \phi_{n}\left(x_{\mathrm{s}}, y_{\mathrm{s}}\right)}{2 S \Lambda_{n} k_{n z}} e^{-\mathrm{j} k_{n z}\left|L_{z}-z_{\mathrm{s}}\right|}\right] \frac{e^{-\mathrm{j} k r}}{2 \pi r} d S_{0},
\end{aligned}
$$

where $p_{\text {in }}$ and $p_{\text {out }}$ are the sound pressure inside and outside the enclosure, respectively; $A_{n}^{\mathrm{i}}$ and

$A_{n}^{\mathrm{r}}$ are coefficients of the $n$th mode which can be obtained by considering the boundary conditions. $\phi_{n}(x, y)=\cos \left(n_{x} \pi / l_{x}\right) \cos \left(n_{y} \pi / l_{y}\right)$ is the mode shape. $k_{n z}=\left[k^{2}-\left(n_{x} \pi / l_{x}\right)^{2}-\left(n_{y} \pi / l_{y}\right)^{2}\right]^{1 / 2}$ is the wave number in the $z$ direction; $k$ is the wave number, $\omega$ is the angular frequency, $S_{0}$ is the opening area, and $\rho_{0}$ is the air density. $\Lambda_{n}$ is a constant depending on $n_{x}$ and $n_{y}\left(\Lambda_{n}=1\right.$ for both $n_{x}$ and $n_{y}=0 ; \Lambda_{n}=0.5$ for only one of $n_{x}$ and $n_{y}=0 ; \Lambda_{n}=0.25$ for neither of $n_{x}$ and $n_{y}=$ $0)$. Finally, $r$ is the distance from the field point $(x, y, z)$ to the integration point $\left(x_{0}, y_{0}, L_{z}\right)$ on the opening area $S_{0}$.

\subsection{Planar virtual sound barrier}

For the PVSB system shown in Fig. 1, it is assumed that the number of error sensors is the same as that of the control sources, i.e., there are $M$ loudspeakers and $M$ error microphones in the system. The signal vector from the error microphones can be expressed as

$$
\mathbf{e}=\mathbf{p}+\mathbf{Z} \mathbf{Q}_{\mathrm{c}}
$$

where $\mathbf{p}$ is the sound pressure vector from the primary source, $\mathbf{Z}$ is an $M \times M$ matrix of the transfer functions from the $M$ secondary loudspeakers to the $M$ error microphones, and $\mathbf{Q}_{\mathrm{c}}$ is the output strength vector. In the following simulations, all transfer functions are obtained with Eqs. (1) and (2) by substituting the specific positions of the sources and microphones.

When the output strength of each secondary loudspeaker is adjusted in response to the error signals at all error microphones, the PVSB system is fully coupled and the transfer function matrix $\mathbf{Z}$ is adopted in the optimization. However when the output strength of each 
secondary loudspeaker is adjusted with only the error signal at the related error microphone, the PVSB system is independent and a diagonal matrix $\mathbf{Z}^{\mathrm{I}}$ is employed. The off-diagonal elements of $\mathbf{Z}^{\mathrm{I}}$ are zero but the diagonal elements of $\mathbf{Z}^{\mathrm{I}}$ are the same as those of $\mathbf{Z}$, where the superscript ${ }^{\mathrm{I}}$ is used to indicate that the system is independent.

For a fully coupled PVSB system, the output strength vector $\mathbf{Q}_{\mathrm{c}}$ is obtained by minimizing the cost function

$$
J=\mathbf{e}^{\mathrm{H}} \mathbf{e}+\beta \mathbf{Q}_{\mathrm{c}}^{\mathrm{H}} \mathbf{Q}_{\mathrm{c}}
$$

where $\beta$ is a positive real weighting factor and the superscript ${ }^{\mathrm{H}}$ denotes the matrix transpose. The gradient of the cost function is:

$$
\nabla J=2 \mathbf{Z}^{\mathrm{H}} \mathbf{e}+2 \beta \mathbf{Q}_{\mathrm{c}} .
$$

However for an independent PVSB system, the cost function is defined for each independent control channel as

$$
J_{i}^{\mathrm{I}}=e_{i}^{*} e_{i}+\beta_{i}^{\mathrm{I}}\left(Q_{c i}^{\mathrm{I}}\right)^{*} Q_{c i}^{\mathrm{I}}, \text { for } i=1,2,3, \ldots, M
$$

The gradient of the cost function of the $i$ th channel is:

$$
\nabla J_{i}^{\mathrm{I}}=2 Z_{i}^{\mathrm{I}} e_{i}+2 \beta_{i}^{\mathrm{I}} Q_{\mathrm{c} i}^{\mathrm{I}}
$$

where $Z_{i}^{\mathrm{I}}, e_{i}, \beta_{i}^{\mathrm{I}}$ and $Q_{\mathrm{c} i}^{\mathrm{I}}$ are the transfer function, error signal, weighting factor and the output strength, respectively, of the $i$ th independent channel. The weighting factor of each channel, $\beta_{i}^{\mathrm{I}}$, can be adjusted separately in the independent PVSB system instead of the single constant value $\beta$ adopted in the fully coupled system. If all the weighting factors equal zero, then the minimization target of the independent PVSB system is the squared sound pressure at each error microphone rather than the summation of the squared sound pressure of all error microphones in the fully coupled system.

Assuming Eqs. (4b) and (5b) equal zero, the optimal output strength can be directly calculated as: 


$$
\begin{aligned}
& \mathbf{Q}_{\mathrm{c}}(\infty)=-\left[\mathbf{Z}^{\mathrm{H}} \mathbf{Z}+\beta \mathbf{I}\right]^{-1} \mathbf{Z}^{\mathrm{H}} \mathbf{p} \\
& \mathbf{Q}_{\mathrm{c}}^{\mathrm{I}}(\infty)=-\left[\left(\mathbf{Z}^{\mathrm{I}}\right)^{\mathrm{H}} \mathbf{Z}+\boldsymbol{\beta}^{\mathrm{I}} \mathbf{I}\right]^{-1}\left(\mathbf{Z}^{\mathrm{I}}\right)^{\mathrm{H}} \mathbf{p},
\end{aligned}
$$

where $\beta$ is a diagonal matrix for the independent PVSB system and the diagonal elements are the weighting factors of all channels. If both $\beta$ and $\beta$ ' equal zero, the output strength can be expressed as $\mathbf{Q}_{\mathrm{c}}(\infty)=\mathbf{Q}_{\mathrm{c}}^{\mathrm{I}}(\infty)=-\mathbf{Z}^{-1} \mathbf{p}$, so the noise reduction performance of the independent PVSB system is the same as that of the fully coupled one.

In practical applications, the cost function minimization is typically implemented by adopting an iteration algorithm. The output strength can be obtained by $[12,13]$

$$
\begin{aligned}
& \mathbf{Q}_{\mathrm{c}}(n+1)=\mathbf{Q}_{\mathrm{c}}(n)-\mu \nabla J \\
& \mathbf{Q}_{\mathrm{c}}^{\mathrm{I}}(n+1)=\mathbf{Q}_{\mathrm{c}}^{\mathrm{I}}(n)-\boldsymbol{\mu}^{\mathrm{I}} \nabla \mathbf{J}^{\mathrm{I}},
\end{aligned}
$$

where $n$ is the iteration number, $\mu$ is the step size for the fully coupled system, $\boldsymbol{\mu}^{\mathrm{I}}$ is a diagonal matrix consisting of the step sizes of $\mu_{i}^{\mathrm{I}}$, and $\nabla \mathbf{J}^{\mathrm{I}}$ is a column vector composed by the gradients of the cost function $\nabla J_{i}^{\mathrm{I}}$. Substituting Eq. (6) into Eq. (7), it can be found that [12]:

$$
\begin{aligned}
& {\left[\mathbf{Q}_{\mathrm{c}}(n+1)-\mathbf{Q}_{\mathrm{c}}(\infty)\right]=\left[\mathbf{I}-2 \mu\left(\mathbf{Z}^{\mathrm{H}} \mathbf{Z}+\beta\right)\right]\left(\mathbf{Q}_{\mathrm{c}}(n)-\mathbf{Q}_{\mathrm{c}}(\infty)\right) \text { and }} \\
& {\left[\mathbf{Q}_{\mathrm{c}}^{\mathrm{I}}(n+1)-\mathbf{Q}_{\mathrm{c}}^{\mathrm{I}}(\infty)\right]=\left\{\mathbf{I}-2 \boldsymbol{\mu}^{\mathrm{I}}\left(\left(\mathbf{Z}^{\mathrm{I}}\right)^{\mathrm{H}} \mathbf{Z}+\boldsymbol{\beta}^{\mathrm{I}}\right]\left[\mathbf{Q}_{\mathrm{c}}^{\mathrm{I}}(n)-\mathbf{Q}_{\mathrm{c}}^{\mathrm{I}}(\infty)\right]\right.}
\end{aligned}
$$

To ensure this iteration equation converges for large $n$, the moduli of all eigenvalues of the matrix in square brackets should be less than unity, which means that the real parts of all the eigenvalues of $\mathbf{Z}^{\mathrm{H}} \mathbf{Z}+\beta$ and $\left(\mathbf{Z}^{\mathrm{I}}\right)^{\mathrm{H}} \mathbf{Z}+\boldsymbol{\beta}^{\mathrm{I}}$ must be positive [12]. For the fully coupled PVSB system, the matrix $\mathbf{Z}^{\mathrm{H}} \mathbf{Z}$ is always positive and symmetrical. When the step size $\mu$ is sufficiently small, $\mathbf{I}-2 \mu\left(\mathbf{Z}^{\mathrm{H}} \mathbf{Z}+\beta\right)$ is less than unity no matter what the value of $\beta$ is. Thus the fully coupled system is guaranteed to be stable when the step size is sufficiently small.

For the independent PVSB system, $\mathbf{Z}^{\mathrm{I}}$ is a diagonal matrix, and the sign and symmetry of 
the matrix $\left(\mathbf{Z}^{\mathrm{I}}\right)^{\mathrm{H}} \mathbf{Z}+\boldsymbol{\beta}^{\mathrm{I}}$ depends on the specific values of all elements. When the weighting factors in $\boldsymbol{\beta}^{\mathrm{I}}$ are sufficiently large, $\left(\mathbf{Z}^{\mathrm{I}}\right)^{\mathrm{H}} \mathbf{Z}+\boldsymbol{\beta}^{\mathrm{I}}$ tends to be the diagonal and positive matrix, $\boldsymbol{\beta}^{\mathrm{I}}$, and the independent PVSB system should also be stable with a small step size matrix $\boldsymbol{\mu}^{\mathrm{I}}$. A sufficient but not necessary condition based on the Gerschgorin circle theorem to ensure the stability of the independent PVSB system was proposed as [12]:

$$
\left|Z_{i i}\right|+\beta_{i}^{\mathrm{I}} /\left|Z_{i i}\right| \geq \sum_{j=1, j \neq i}^{M}\left|Z_{i j}\right| \text { for all } i,
$$

where $Z_{i j}$ is the transfer function from the $i$ th secondary loudspeaker to the $j$ th error microphone. If the system is in free space, $Z_{i j}$ can be calculated using the Green's function $Z_{i j}=e^{-\mathrm{j} k r_{i j}} / 4 \pi r_{i j}$, where $r_{i j}=\sqrt{\left(x_{j}-x_{i}\right)^{2}+\left(y_{j}-y_{i}\right)^{2}+\left(z_{j}-z_{i}\right)^{2}},\left(x_{i}, y_{i}, z_{i}\right)$ and $\left(x_{j}, y_{j}, z_{j}\right)$ are the coordinates of the $i$ th secondary loudspeaker and the $j$ th error microphone, respectively. If the distance between each secondary loudspeaker and its corresponding error microphone is $d$, then Eq. (9) can be rewritten as:

$$
\frac{1}{d}+\beta_{i}^{\mathrm{I}} d \geq \sum_{j=1, j \neq i}^{M} \frac{1}{\sqrt{\left(x_{j}-x_{i}\right)^{2}+\left(y_{j}-y_{i}\right)^{2}+d^{2}}} \text { for all } i
$$

When all the weighting factors $\beta_{i}^{\mathrm{I}}$ are set to zero and the term of $d^{2}$ on the right-hand side is neglected (this means the minimum distance between two independent single channel systems is larger than the distance from the secondary loudspeaker to the corresponding error sensor), Eq. (10) can be simplified as:

$$
\frac{1}{d} \geq \max \left[\left.\sum_{j=1, j \neq i}^{M} \frac{1}{\sqrt{\left(x_{j}-x_{i}\right)^{2}+\left(y_{j}-y_{i}\right)^{2}}}\right|_{i=1, \cdots, M}\right] .
$$

Equation (11) indicates that the system stability can be ensured at any frequency when the distance between the secondary loudspeaker and its corresponding error microphone in an independent system is sufficiently small. If the covering area of the independent PVSB system is fixed, then increasing the number of channels enhances the value of the right-hand 
side of Eq. (11) and therefore a smaller distance $d$ is required to ensure the stability. In other words, it can be predicted that the stability of the independent PVSB can be improved by reducing the distance between the planes of the secondary loudspeakers and error microphones or by reducing the number of channels at the cost of the loss of noise reduction performance.

Based on the analysis above, it is found that the stability of an independent PVSB system is not as good as that of a fully coupled one. However, if the stability requirement can be met, then the noise control performance of the independent PVSB system in free space should be the same as that of the fully coupled system when the weighting factors equal zero. The stability and convergence behaviors of the independent PVSB system at the enclosure opening will be further investigated in the following simulations and experiments, where the radiated sound power is approximated using the squared sound pressure in far field as:

$$
L_{\mathrm{w}}=10 \log _{10}\left(\frac{\sum_{m=1}^{N_{o}} p_{m}^{2} \Delta S_{m}}{2 \rho_{0} c_{0} W_{\text {ref }}}\right),
$$

where $N_{e}$ is the number of evaluation points, $p_{m}$ and $\Delta S_{m}$ are the sound pressure and the covering area of the $m$ th evaluation point, $c_{0}$ is the sound speed, and $W_{\text {ref }}$ is the reference sound power $10^{-12} \mathrm{~W}$.

\section{Simulations}

The enclosure dimensions in the simulation are chosen as $L_{x}=0.432 \mathrm{~m}, L_{y}=0.670 \mathrm{~m}$ and $L_{z}=0.598 \mathrm{~m}$. The primary source is placed at $(0.12,0.12,0.16) \mathrm{m}$ in order to excite the as many enclosure modes as possible with a strength of $1 \times 10^{-4} \mathrm{~m}^{3} / \mathrm{s}$. As shown in Fig. 1, six secondary loudspeakers are adopted in a horizontal plane $L_{\mathrm{c}}=0.498 \mathrm{~m}$, which is $0.1 \mathrm{~m}$ below the enclosure opening. The one nearest to the $z$ axis locates at $(0.108,0.1117,0.498) \mathrm{m}$ and the intervals in $x$ and $y$ axes are $0.216 \mathrm{~m}$ and $0.223 \mathrm{~m}$. Six error microphones are placed at the 
opening plane $z=0.598 \mathrm{~m}$ with the same $x-y$ coordinates as the secondary loudspeakers. A semi-spherical surface with a radius of $10 \mathrm{~m}$ centered at the opening is chosen for noise reduction performance evaluation, and the sum of the squared sound pressure at 380 intersection points of evenly distributed longitude and latitude lines on the surface are calculated to approximate the sound power.

Assuming that the PVSB systems are stable, the optimal output to the secondary loudspeakers can be directly calculated based on Eq. (6). The obtained sound power with and without the PVSB system is shown in Fig. 2. As deduced from Eq. (6), the same noise reduction is achieved for both fully coupled and independent systems when all weighting factors are zero. The negative noise reduction at $170 \mathrm{~Hz}$ and $305 \mathrm{~Hz}$ with zero weighting factors is due to the arrangement of error microphones, which leads to a mismatch between the sound power and the sum of squared sound pressures at the error microphones.

Increasing the weighting factor increases the constraint on the output strength in the practical applications at the cost of noise reduction performance degradation $[10,11]$. Fig. 2 shows that when the weighting factors are increased from zero to $2.5 \times 10^{7}$, the performance of both PVSB systems is degraded and the noise reduction of the independent PVSB system is poorer than that of the fully coupled one at most frequencies. As shown in Eq. (6b), the diagonal matrix $\mathbf{Z}^{\mathrm{I}}$ instead of the transfer function matrix $\mathbf{Z}$ is adopted in the independent PVSB system to obtain the optimal output strength, and the sound pressure contributed by other secondary loudspeakers is excluded in the optimization of each independent single channel system. This is the reason for the poorer noise reduction performance of the independent PVSB system. Fig. 2 also shows that for all configurations, the noise is reduced significantly at the peak frequencies of the sound power of the primary noise, such as $105 \mathrm{~Hz}$, $280 \mathrm{~Hz}, 415 \mathrm{~Hz}$ and $485 \mathrm{~Hz}$. 


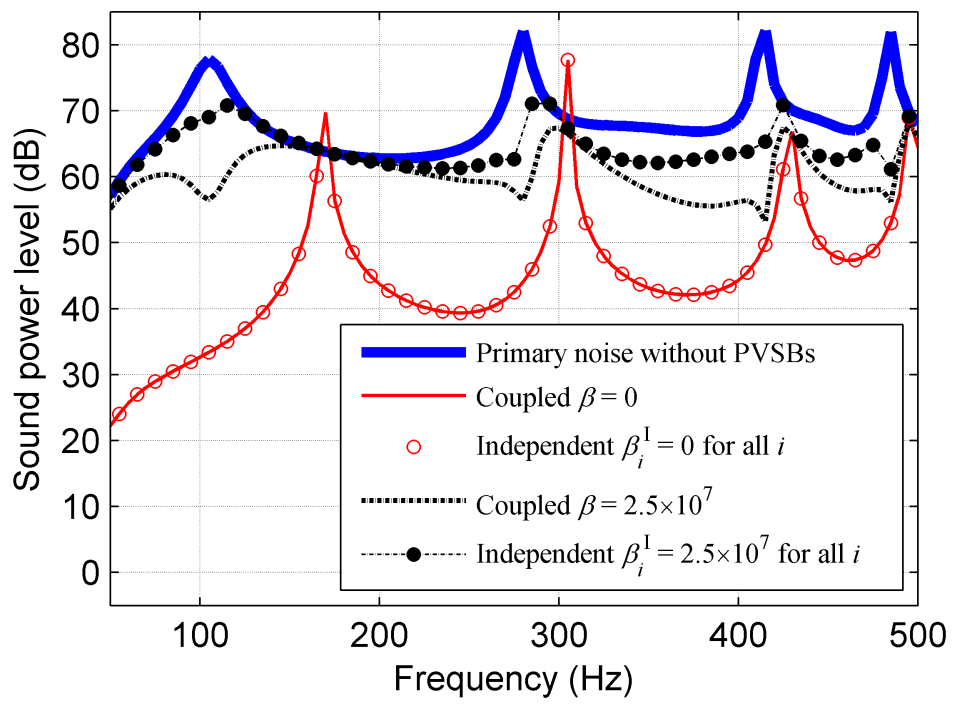

Fig. 2. Radiated sound power with and without six-channel PVSB systems using different weighting factors.

\subsection{Stability of the independent PVSB system}

The real part of all the eigenvalues of the matrix $\left(\mathbf{Z}^{\mathrm{I}}\right)^{\mathrm{H}} \mathbf{Z}+\boldsymbol{\beta}^{\mathrm{I}}$ must be positive in order to maintain the stability of the independent PVSB system [12]. When the sign of the minimum is positive, the real parts of all the eigenvalues are positive and the PVSB system is stable. The sign of the real part of the minimum of the eigenvalues of the six-channel independent PVSB system is shown in Fig. 3. It is clear that the system stability requirement cannot be satisfied at frequencies around $130 \mathrm{~Hz}$ and $290 \mathrm{~Hz}$ and above $380 \mathrm{~Hz}$ when all the weighting factors equal zero, because the minimum of the eigenvalues is negative at these frequencies. 


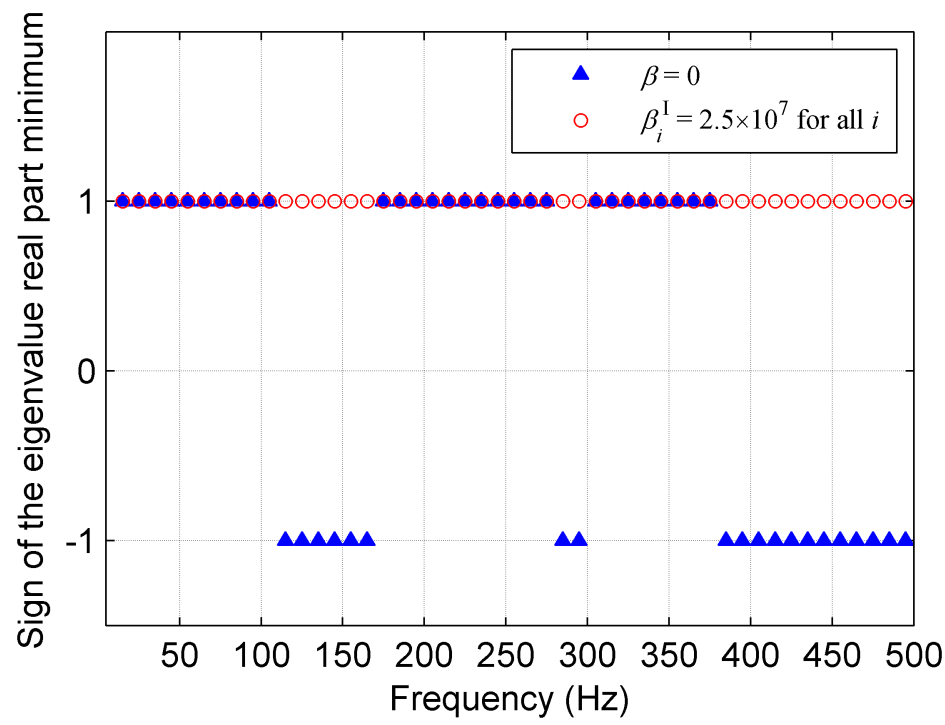

Fig. 3. Sign of the minimum of the eigenvalues' real part when the distance between the secondary loudspeaker plane and error sensor plane is $0.1 \mathrm{~m}$ for the six-channel independent PVSB system.

If all $\beta_{i}^{\mathrm{I}}$ are sufficiently large, the matrix $\left(\mathbf{Z}^{\mathrm{I}}\right)^{\mathrm{H}} \mathbf{Z}+\boldsymbol{\beta}^{\mathrm{I}}$ can be approximately by $\boldsymbol{\beta}^{\mathrm{I}}$. The eigenvalues of $\left(\mathbf{Z}^{\mathrm{I}}\right)^{\mathrm{H}} \mathbf{Z}+\boldsymbol{\beta}^{\mathrm{I}}$ are the values of the weighting factors $\beta_{i}{ }^{\mathrm{I}}$. Because the weighting factors are all positive real numbers, the stability of the independent PVSB system can be improved by increasing the weighting factors. As shown in Fig. 3, when the weighting factors are increased to $2.5 \times 10^{7}$, the sign of the minimum of the real part of eigenvalue is positive at all frequencies below $500 \mathrm{~Hz}$. The corresponding noise reduction performance has already been presented in Fig. 2.

The arrangement of the secondary loudspeakers and the error microphones is an important factor for determining the transfer function matrix $\mathbf{Z}$. Figure 4 shows the noise reduction performance of the independent PVSB systems with different numbers of channels when the secondary loudspeaker plane is located at $L_{\mathrm{c}}=0.498 \mathrm{~m}$ and all the weighting factors equal zero. The missing (empty or blank) sections in the curves correspond to the frequencies at which the system cannot meet the stability requirement. For the two-channel system, the secondary loudspeakers are located at $\left(\begin{array}{llll}0.216 & 0.1675 & 0.498\end{array}\right) \mathrm{m}$ and $\left(\begin{array}{lll}0.216 & 0.5025 & 0.498\end{array}\right) \mathrm{m}$. For the fifteen-channel PVSB system, secondary loudspeakers are evenly distributed with 
intervals of $0.149 \mathrm{~m}$ and $0.134 \mathrm{~m}$ along the $x$ and $y$ directions. The error microphones are located on the opening surface above the corresponding secondary loudspeakers.

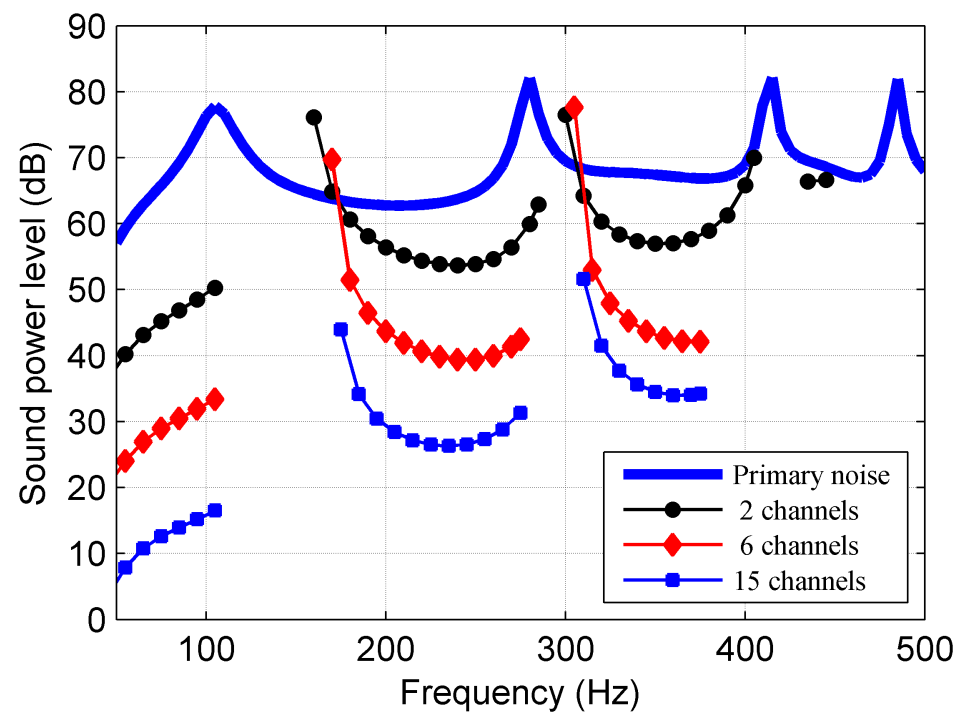

Fig. 4. Radiated sound power of the independent PVSB systems with different numbers of channels when the secondary loudspeaker plane is $0.1 \mathrm{~m}$ below the opening and the weighting factors are zero.

Figure 4 shows that the noise reduction increases with the number of channels; however, there is some extension of the unstable frequency range. The results agree with the analyses for the PVSB system in free space based on Eq. (11). A compromise has to be made between the noise reduction performance and the system stability margin when determining the number of channels for independent PVSB systems. It is also notable that when the number of channels increases from 6 to 15 , the noise reduction at $170 \mathrm{~Hz}$ and $305 \mathrm{~Hz}$ increases from negative to positive. If the error microphone arrangement in six-channel systems and the secondary loudspeaker arrangement in fifteen-channel systems are adopted, the noise reduction at $170 \mathrm{~Hz}$ and $305 \mathrm{~Hz}$ is still zero for the fully coupled system with a zero weighting factor. This confirms the deduction from Fig. 2 that the arrangement of error microphones induces the negative noise reduction of six-channel systems at $170 \mathrm{~Hz}$ and 305 $\mathrm{Hz}$. 
The variation in performance of the six-channel independent PVSB systems with the interval between the secondary loudspeaker plane and the opening is plotted in Fig. 5. It can be seen that the range of the unstable frequency is narrowed and the noise reduction value decreases when the plane of the secondary loudspeakers moves closer to the opening. The percentage of unstable frequencies drops from $40 \%$ to nearly zero when the interval between the planes of secondary loudspeakers and the error microphones decreases from $0.05 \mathrm{~m}$ to $0.01 \mathrm{~m}$, but the noise reduction also drops to around $10 \mathrm{~dB}$ at most frequencies.

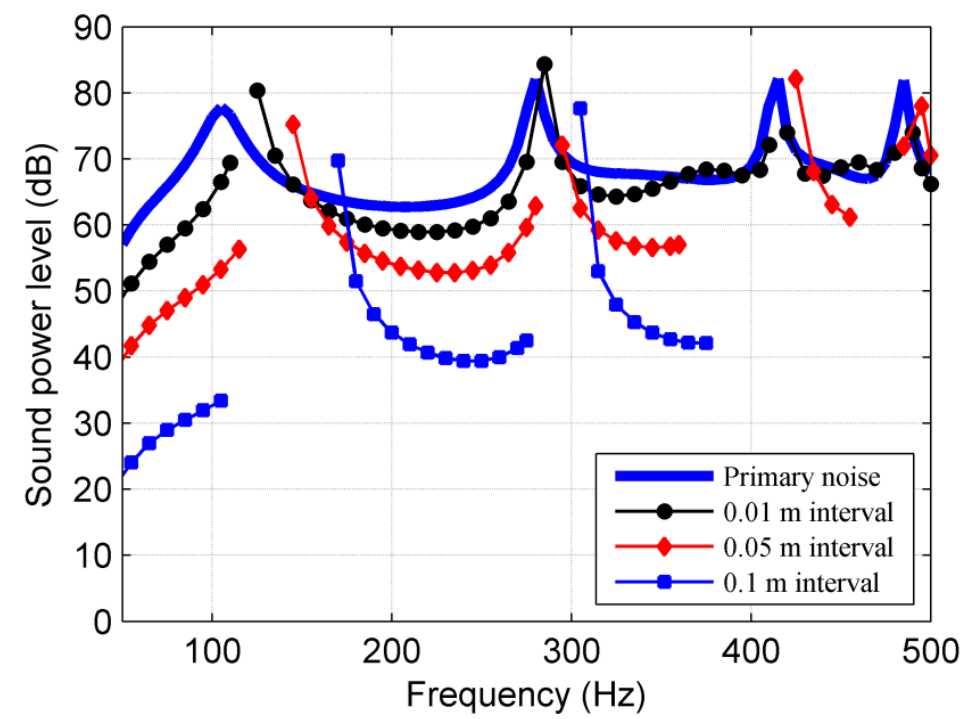

Fig. 5. Radiated sound power of the six-channel independent PVSBsystems with different secondary loudspeaker locations when the weighting factors are zero.

Based on the analysis above, it can be concluded that the independent PVSB system is less stable, because it becomes unstable at some frequencies while the fully coupled system remains stable at all frequencies if the weighting factors are zero. However the stability of the independent system can be improved by increasing the weighting factors. The noise reduction performance might be reduced because the constraint of the output strength becomes stronger. The stability of the independent system can also be improved by reducing the interval between secondary loudspeakers and error microphones and by reducing the number of control channels. The reason is that the influence of the non-collocated secondary source 
becomes smaller when the number of non-collocated loudspeakers decreases and when the distance to the collocated loudspeaker becomes smaller for a given error microphone.

\subsection{Convergence due to step size}

In practical applications, the output of secondary loudspeakers is obtained through adaptive iterations rather than by adopting the calculated optimal value directly from Eq. (6). Equation (8) shows that PVSB systems become divergent in iterative updating if the step size is not sufficiently small, even though the stability requirement (the real part of all eigenvalues

of the matrix $\left(\mathbf{Z}^{\mathrm{I}}\right)^{\mathrm{H}} \mathbf{Z}+\boldsymbol{\beta}^{\mathrm{I}}$ must be positive) is satisfied.

The convergence curve of the radiated sound power at a randomly chosen frequency, 100 $\mathrm{Hz}$, is presented in Fig. 6 as an example, where it is clear that both the fully coupled and independent PVSB systems can converge when the step size is sufficiently small. Figure 6 also shows that the fully coupled PVSB becomes divergent while the independent system remains convergent when the step size increases to a certain value. The upper bounds of the step size for convergence of the independent PVSB systems are typically higher than those of the fully coupled systems. This is reasonable as implied in Eq. (8) because the amount of adjustment of the fully coupled PVSB systems depends on the product of the step size and the summation of all error signals while the amount of adjustment of the independent systems depends only on the product of the step size and the one error signal. 


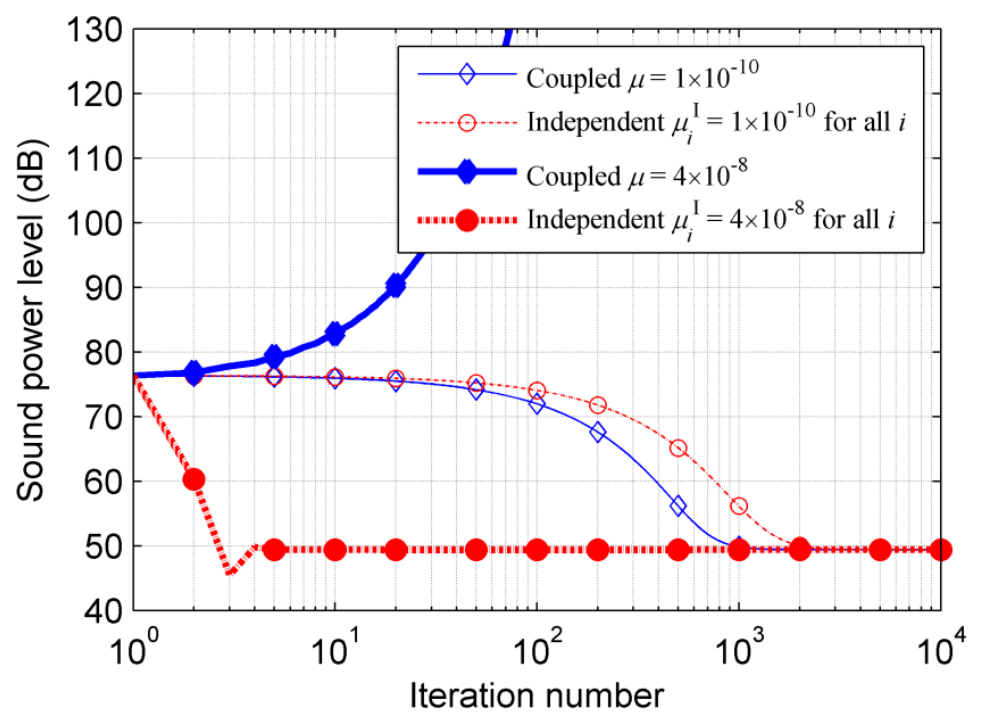

(a)

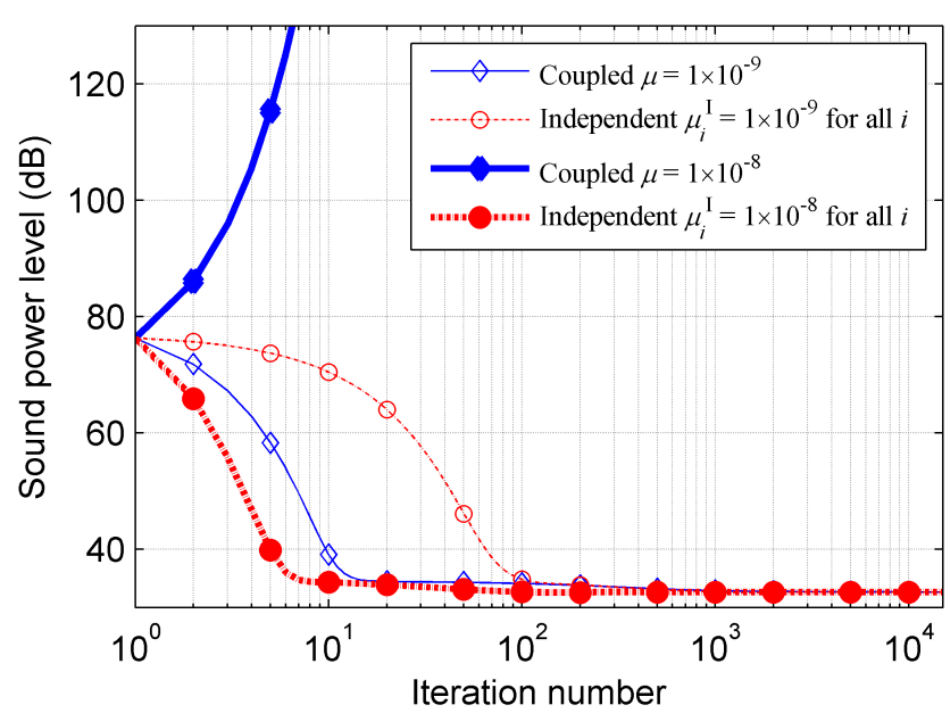

(b)

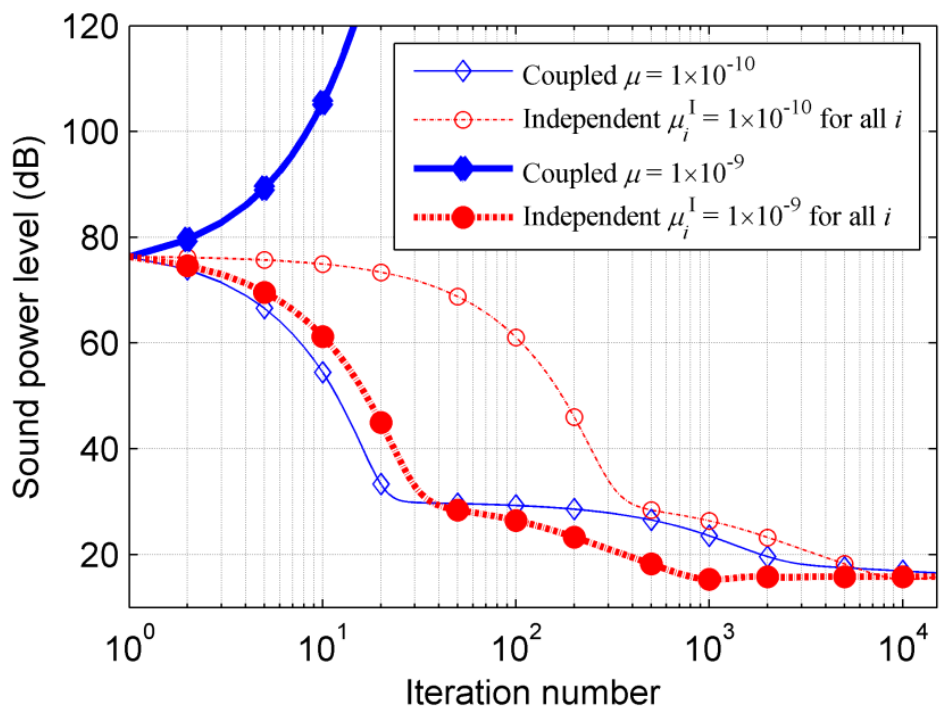

(c) 
Fig. 6. Convergence curves of the PVSB systems at $100 \mathrm{~Hz}$ with weighting factors of zero (a) two-channel systems (b) six-channel systems (c) fifteen-channel systems.

The upper bound of the step size for convergence at different frequencies is calculated and plotted in Fig. 7 for the six-channel systems with the weighting factor of zero. Compared with the sound power level shown in Fig. 2, it is interesting that the lower upper bound of the step size of both PVSB systems corresponds to a higher noise reduction value in general. For example, the noise reduction is larger while the upper bound of the step size is smaller when the frequency increases from zero to $100 \mathrm{~Hz}$. It can also be found that the upper bound of the step size of the independent PVSB system is higher than that of the fully coupled system at most frequencies.

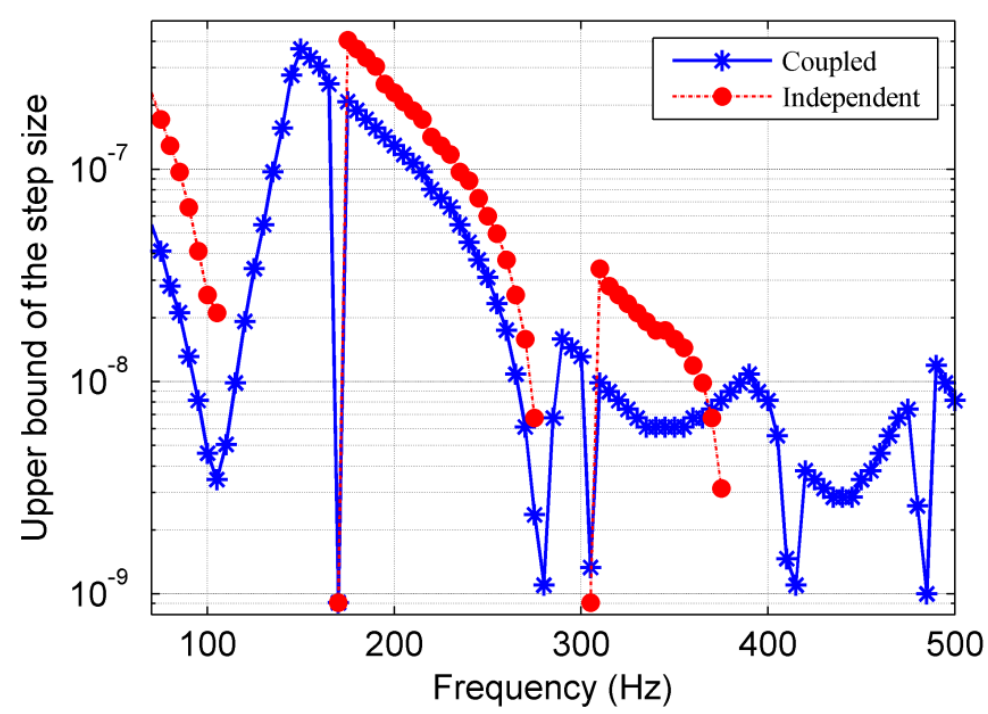

Fig. 7. Upper bound of the step size to ensure convergence for the six-channel systems with weighting factors of zero.

The convergence behavior of the sound power level during the adaptation process at three frequencies, $100 \mathrm{~Hz}, 200 \mathrm{~Hz}$ and $310 \mathrm{~Hz}$, is presented in Fig. 8, where the weighting factors are zero and the step size for convergence is manually set as high as possible for convergence. (As shown in Fig. 7, the independent system cannot converge at $300 \mathrm{~Hz}$, whatever the step size is, and therefore the nearby value of $310 \mathrm{~Hz}$ is chosen as frequency for 
investigation.) Fig. 8 shows that both fully coupled and independent PVSB systems converge to the same value at a given value of iteration and the convergence rate of the independent PVSB system is faster because it has an upper bound of the step size as shown in Fig. 7.

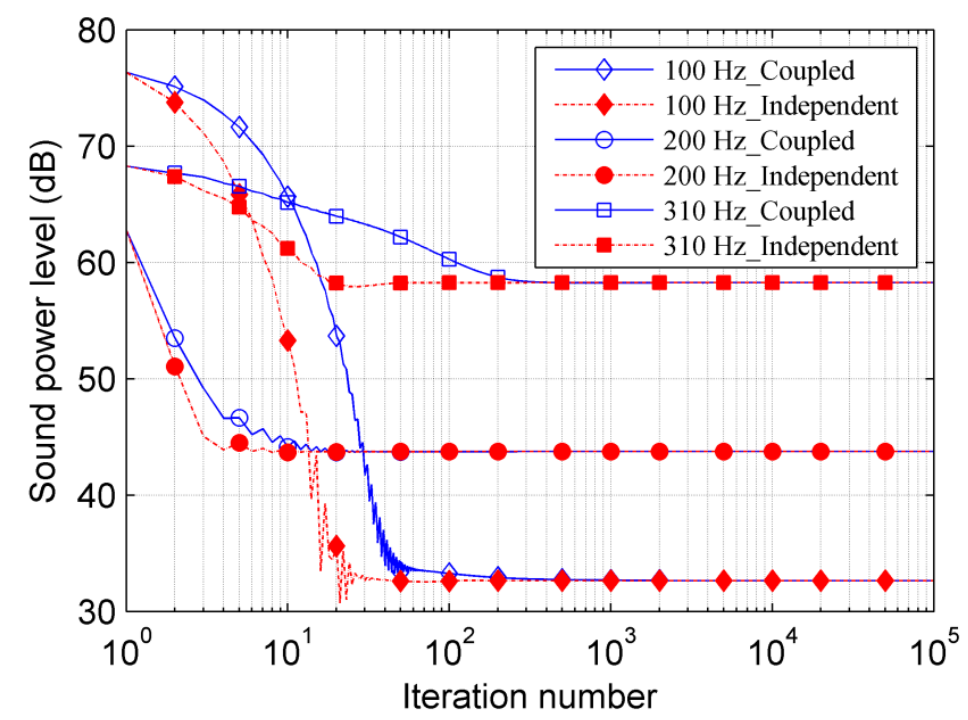

Fig. 8. Convergence curves of the six-channel PVSB systems with the maximum step size and weighting factors of zero.

The simulation results in this section showed that the independent PVSB system can provide the same noise reduction as the fully coupled system when the system stability is ensured, and that it has a larger upper bound of the step size for convergence which means that its convergence speed is faster than that of the fully coupled system.

\section{Experiments}

The experiments were conducted in an anechoic room in the Institute of Acoustics at Nanjing University. As shown in Fig. 9, a plexiglass box with one opening is baffled by a large wooden plate. The inner dimensions of the plexiglass box are $0.432 \mathrm{~m} \times 0.670 \mathrm{~m} \times$ $0.598 \mathrm{~m}$ and the wall thickness is $0.04 \mathrm{~m}$. The transmission loss of the plexiglass walls at 100 $\mathrm{Hz}$ is approximately $32 \mathrm{~dB}$ according to the mass law considering its density of $1200 \mathrm{~kg} / \mathrm{m}^{3}$, so the sound transmission through the walls can be neglected. A semi-spherical frame 
centered at the center of the opening is placed at the plate and 10 microphones are installed on the frame with a $1.5 \mathrm{~m}$ radius to measure the sound power radiated through the opening according to the standard [19].

The primary noise source is located in one corner of the plexiglass box and the virtual sound barrier is composed by 6 loudspeakers and 6 microphones evenly distributed on a plane $0.1 \mathrm{~m}$ below the opening and the opening plane, as shown in Fig. 1. All the primary and secondary loudspeakers are the same, and each is custom made by assembling a 2 inch loudspeaker unit (Peerless, Type: P830983) into a closed plexiglass box (dimensions: 68 $\mathrm{mm} \times 68 \mathrm{~mm} \times 63 \mathrm{~mm}$ ). A commercial active noise controller (Antysound Tiger ANC-II) embedded with the filtered-x LMS algorithm is used for control and a multi-channel analyzer (B\&K PULSE 3560D) is used for data analysis. The electrical signal driving the primary source is also fed to the controller as the reference and all the weighting factors are set to zero. The experiments were conducted for a number of single frequencies in the range between 70 $\mathrm{Hz}$ and $500 \mathrm{~Hz}$ with an interval of $10 \mathrm{~Hz}$.

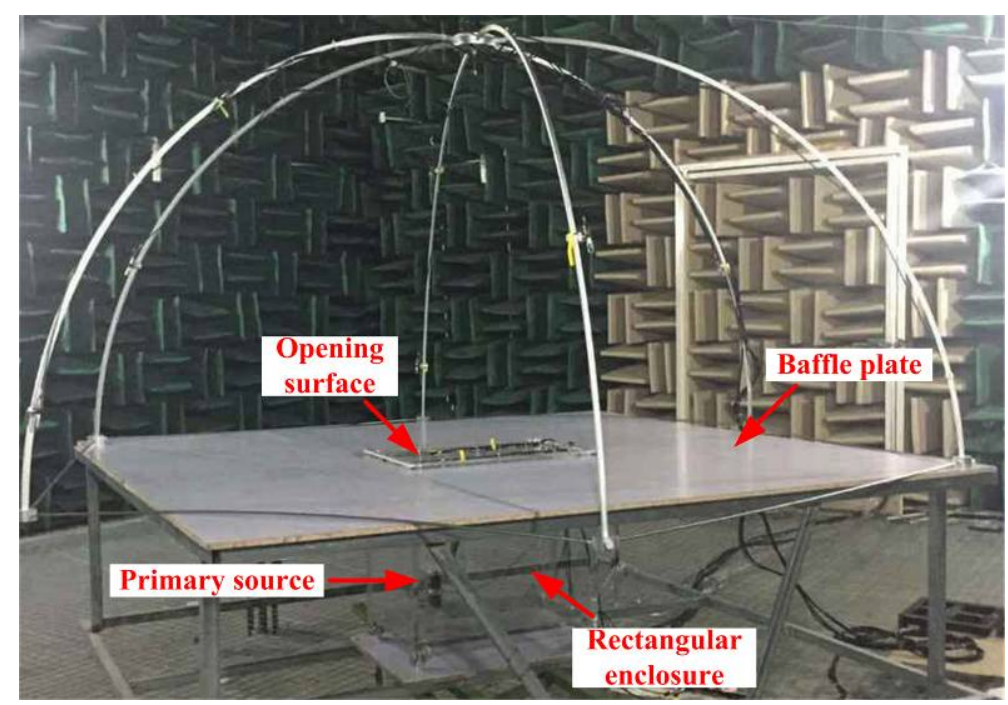

(a) 


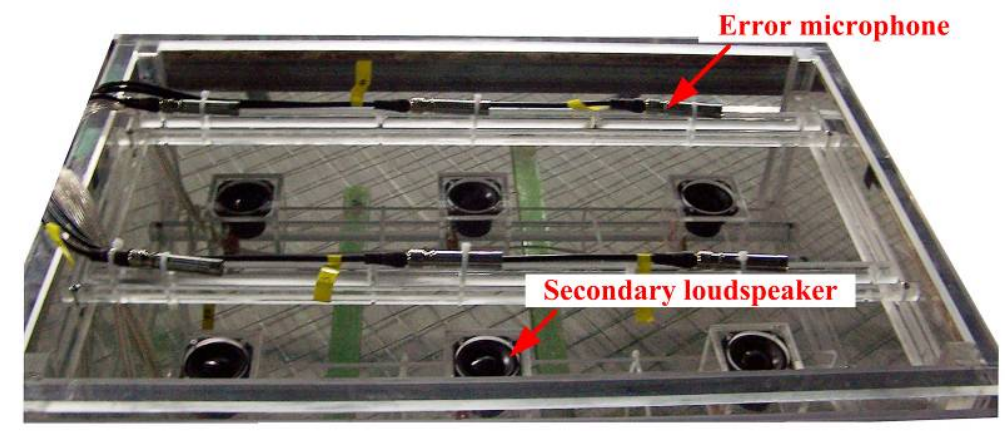

(b)

Fig. 9. Experimental setup (a) the system overview, and (b) configuration of the PVSB system.

The noise reduction performance when the secondary loudspeaker plane is placed at $L_{\mathrm{c}}=$ $0.498 \mathrm{~m}(0.1 \mathrm{~m}$ below the opening $)$ is presented in Fig. 10 , where the trend of the measured noise reduction of the fully coupled PVSB system is almost the same as the simulation results (The simulated noise reduction of the fully coupled and independent PVSB systems is obtained from Fig. 2 and Fig. 4 respectively by subtracting the sound power levels of the primary source and the controlled sound field with zero weighting factors.) except a little frequency shift. Figure10 also shows that the measured noise reduction of the independent system is nearly the same as that of the fully coupled one at the frequencies marked with solid circles. However the system is unstable in the frequency range of $110 \mathrm{~Hz} \sim 160 \mathrm{~Hz}, 280$ $\mathrm{Hz} \sim 300 \mathrm{~Hz}$ and $380 \mathrm{~Hz} \sim 500 \mathrm{~Hz}$ as predicted by the numerical simulations. 


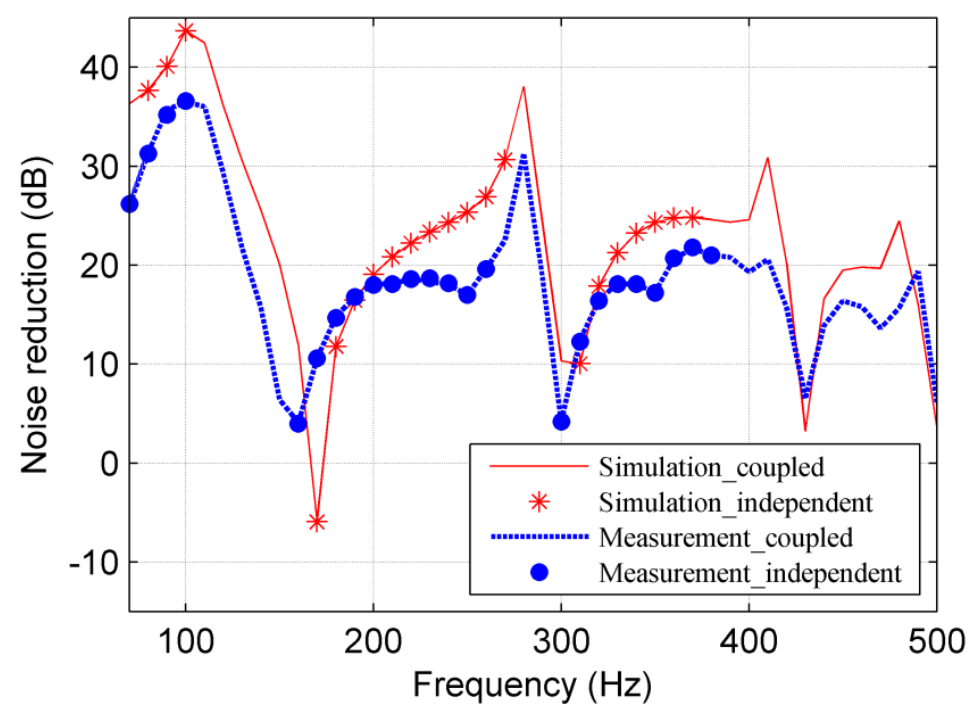

Fig. 10. Comparison between the simulated and measured sound power reduction with the secondary loudspeaker plane $0.1 \mathrm{~m}$ below the opening.

The measured noise reduction performance of the two-channel systems is presented in Fig. 11 (a) when the secondary loudspeaker plane is $0.1 \mathrm{~m}$ below the opening. Fig. 11(a) shows that the two-channel independent PVSB is unstable in the frequency range of 110 $\mathrm{Hz} \sim 140 \mathrm{~Hz}$ and $450 \mathrm{~Hz} \sim 470 \mathrm{~Hz}$, and this frequency range is narrower compared to that of the six-channel independent system. Fig. 11(a) also shows that the noise reduction becomes smaller when the number of system channels decreases.

The measured noise reduction performance when the secondary loudspeaker plane is placed at different heights is presented in Fig. 11 (b). Fig. 11(b) shows that if the secondary loudspeaker plane is $0.05 \mathrm{~m}$ below the opening, the independent PVSB becomes stable at all frequencies except $390 \mathrm{~Hz}, 460 \mathrm{~Hz}$ and $470 \mathrm{~Hz}$. If the secondary loudspeaker plane is $0.01 \mathrm{~m}$ below the opening, the independent PVSB becomes stable at all frequencies below $500 \mathrm{~Hz}$. Fig. 11(b) also shows that if the height of secondary loudspeaker plane is given, the noise reduction performance of the independent PVSB system is the same as that of the fully coupled system because all the weighting factors are zero. However, the value of the noise reduction becomes smaller when the secondary loudspeaker plane becomes closer to the opening. 


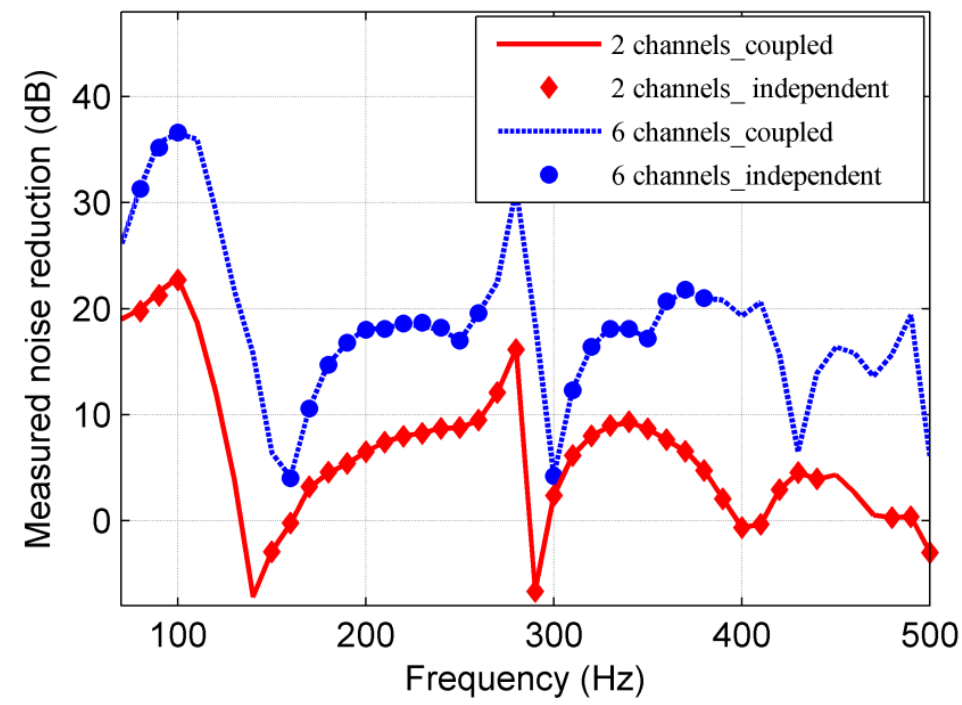

(a)

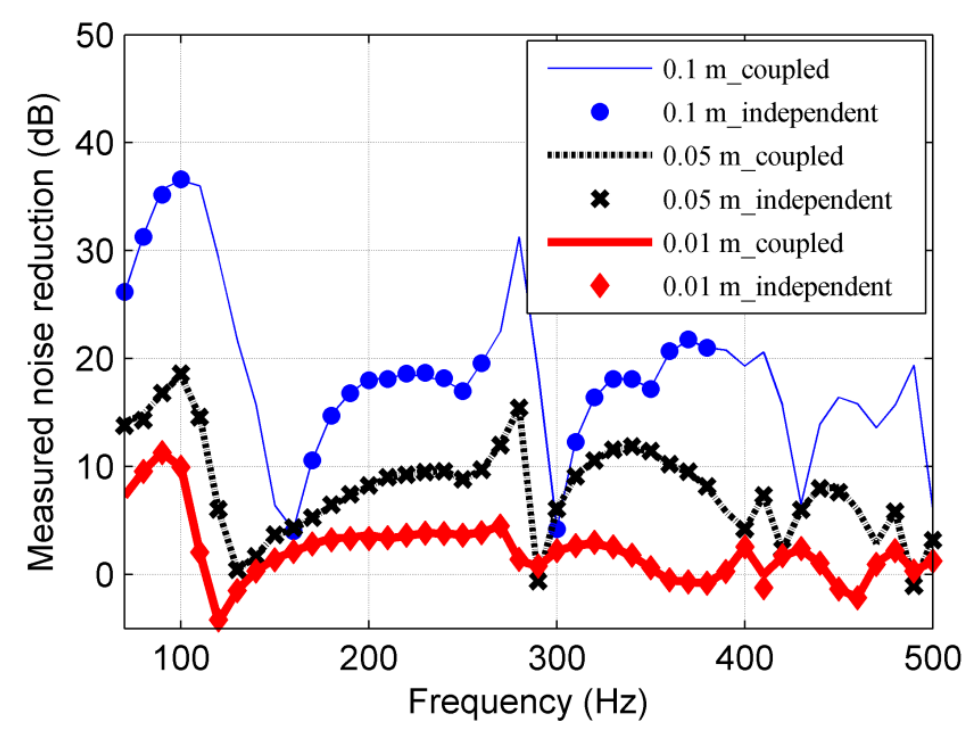

(b)

Fig. 11. Measured sound power reduction for (a) two-channel and six-channel systems with the secondary loudspeakers $0.1 \mathrm{~m}$ below the opening (b) six-channel systems with the secondary loudspeaker plane at different heights.

The upper bound of the step size to ensure system convergence for different PVSB systems is plotted in Fig. 12, where the measured upper bound of the step size of the independent PVSB system is confirmed to be higher than that of the fully-coupled system. The reason for the difference in amplitude between Fig. 12 and Fig. 7 is that peripheral circuits such as the preamplifier for the microphones and the power amplifier for the 
loudspeakers are used in the experiments while the amplification coefficients for all these circuits are assumed to be 1 in the numerical simulations.

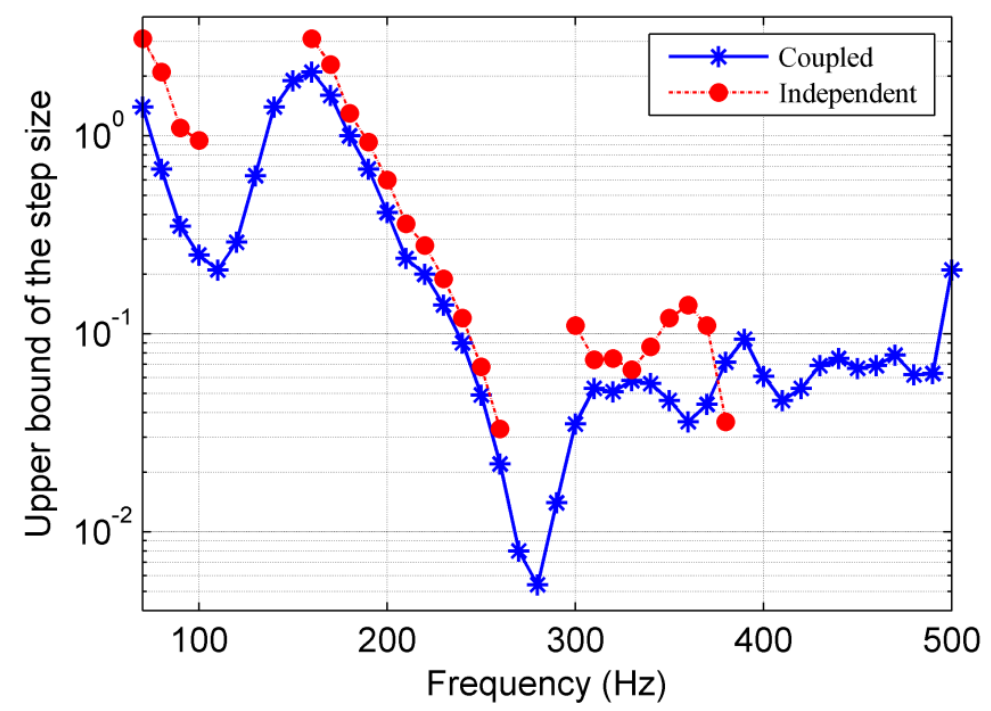

(a)

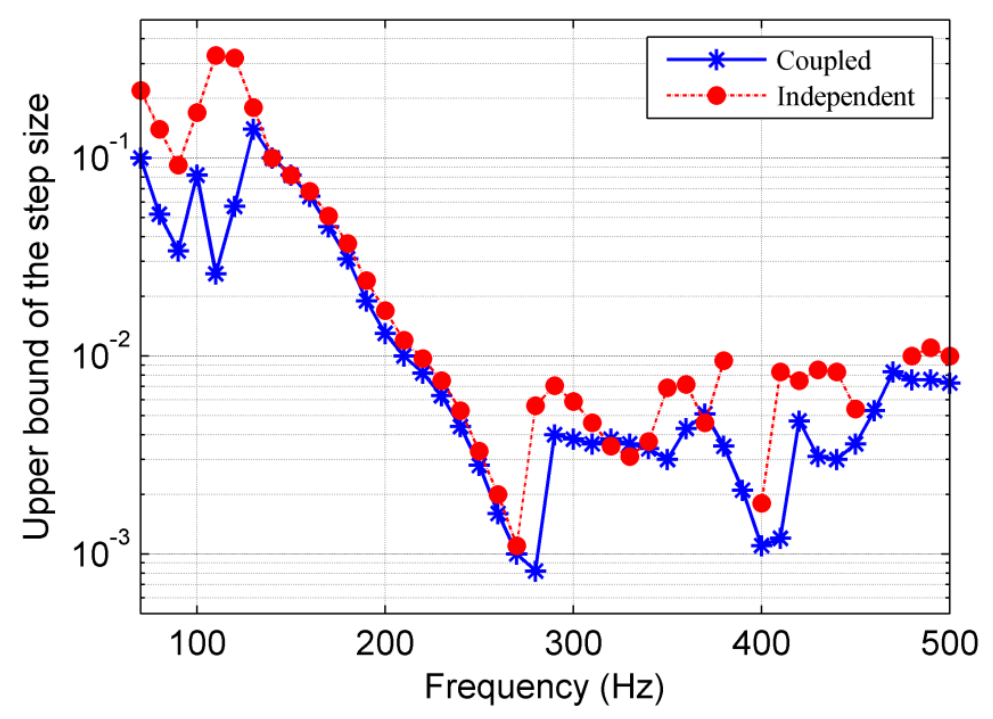

(b) 


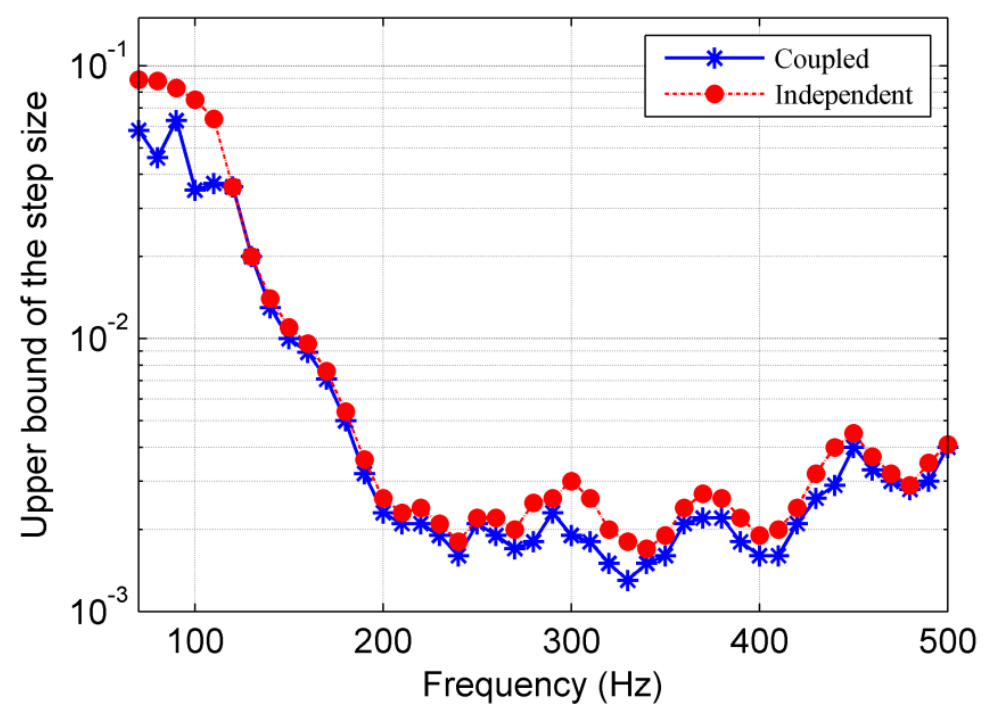

(c)

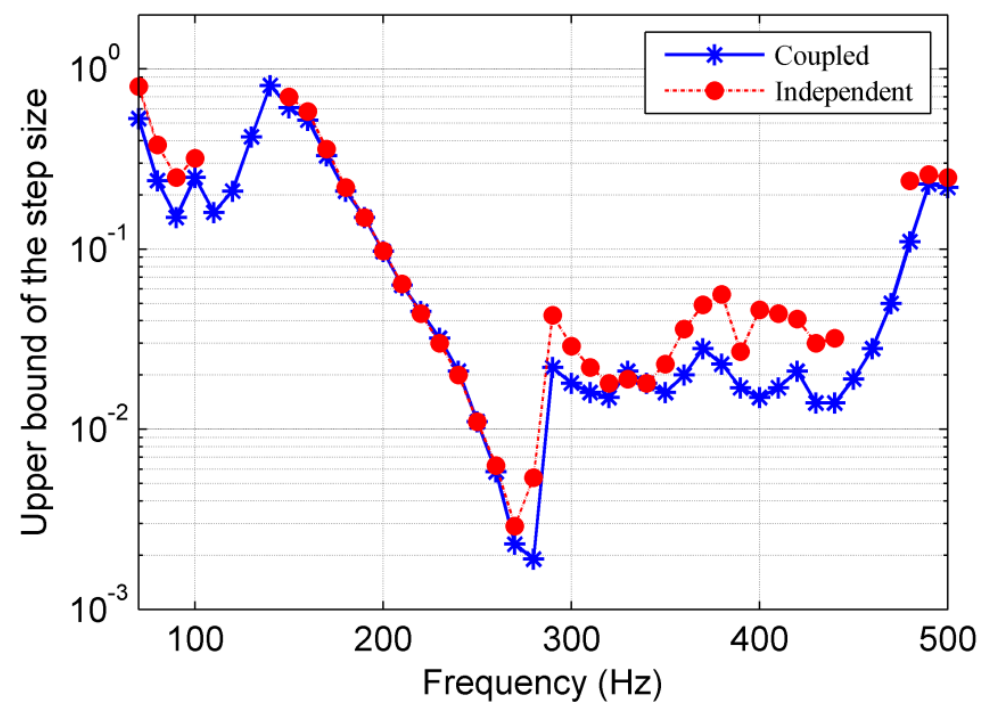

(d)

Fig. 12. Measured upper bound of the step size to ensure system convergence when the secondary loudspeaker plane is located with different intervals to the opening (a) six-channel systems with a $0.1 \mathrm{~m}$ interval (b) six-channel systems with a $0.05 \mathrm{~m}$ interval (c) six-channel systems with a $0.01 \mathrm{~m}$ interval (d) two-channel systems with a $0.1 \mathrm{~m}$ interval.

\section{Conclusions}

The performance of feedforward planar virtual sound barrier (PVSB) systems with fully coupled and independent configurations was investigated based on the analytical model of sound radiation from a monopole inside a rectangular enclosure with one opening. It is found 
that the independent system with no control output constraint becomes inherently unstable at some frequencies because the output strength of each secondary loudspeaker is updated only according to its collocated error microphone; however its stability can be improved at the cost of smaller noise reduction by constraining the output strength and reducing the influence from non-collocated secondary loudspeakers such as reducing the number of channels and the distance between secondary loudspeakers and error microphones. When the system is inherently stable and there is no constraint on the output strength, the independent system can provide the same noise reduction as the fully coupled one but with faster convergence speed.

\section{Acknowledgements}

This research was supported under the Australian Research Council's Linkage Projects funding scheme (LP140100987) and by the National Science Foundation of China (11474163). The authors would also like to thank Ms Xue Jinpei for her help in the experiment.

\section{References}

[1] Qiu X, Li N, Chen G. Feasibility study of developing practical virtual sound barrier system. In: Proceedings of the12th International Congress on Sound and Vibration, Lisbon, Portugal; 2005.

[2] Zou H, Qiu X, Lu J, Niu F. A preliminary experimental study on virtual sound barrier system. J Sound Vib 2007;307:379-85.

[3] Epain N, Friot E. Active control of sound inside a sphere via control of the acoustic pressure at the boundary surface. J Sound Vib 2007;299 2007:587-604.

[4] Zou H. A study on virtual sound barrier system in frequency domain. PhD Thesis, Nanjing University; 2007.

[5] Guo J, Pan J. Further investigation on actively created quiet zones by multiple control sources in free space. J Acoust Soc Am 1997;102:3050-3. 
[6] Guo J, Pan J. Actively created quiet zones for broadband noise using multiple control sources and error microphones. J Acoust Soc Am 1999;105: 2294-303.

[7] Murao T, Nishimura M. Basic study on active acoustic shielding. J Environ Eng 2012;7:76-91.

[8] Ise S. The boundary surface control principle and its applications. IEICE Trans Fundam 2005;E88-A:1656-64.

[9] Wang S, Tao J, Qiu X. Active control of transformer noise radiated outside a three-dimensional building with one opening. Proceedings of the 21st International Congress on Sound and Vibration, Beijing, China; 2014.

[10] Elliott SJ, Boucher CC, Nelson PA, The Behavior of a Multiple Channel Active Control System. IEEE Trans Signal Process 1992;40:1041-52.

[11] Elliott SJ, Signal processing for active control. 1st edition. Academic Press; 2001, p.194-96.

[12] Elliott SJ, Boucher CC. Interaction between multiple feedforward active control systems. IEEE Trans Speech Audio Process 1994;2:521-30.

[13] Cordioli JA, Hansen CH, Li X, Qiu X. Numerical evaluation of a decentralised feedforward active control system for electrical transformer noise. Int J Acoust Vib 2002;7:100-4.

[14] Yu H, Chen K, Sang Z, Dai H. A stability analysis of cluster active control system of sinusoidal sound in free space. Proceedings of the 43rd Proceedings of Inter-noise, Melbourne, Australia; 2014.

[15] Lebouche E, Micheau P, Berry A, L'Espérance A. A stability analysis of a decentralized adaptive feedback active control system of sinusoidal sound in free space. J Acoust Soc Am 2002;111:189-99.

[16] Grosdidier P, Morari M. Interaction measures for systems under decentralized control. Automatica $1986 ; 22: 309-19$.

[17] Zhang L, Tao J, Qiu X. Performance analysis of decentralized multi-channel feedback systems for active noise control in free space. Appl Acoust 2013;74:181-8.

[18] Wang S, Tao J, Qiu X. Performance of a planar virtual sound barrier at the baffled opening of a rectangular cavity. J Acoust Soc Am 2015;138:2836-47.

[19] International Organization for Standardization (ISO). Acoustics - Determination of sound power 
levels and sound energy levels of noise sources with sound pressure - precision methods for anechoic rooms and hemi-anechoic rooms. ISO 3745; 2012. 


\section{Collected Figure Captions}

Fig. 1. A monopole sound source inside a rectangular enclosure with a baffled opening.

Fig. 2. Radiated sound power with and without six-channel PVSB systems using different weighting factors.

Fig. 3. Sign of the minimum of the eigenvalues' real part when the distance between the secondary loudspeaker plane and error sensor plane is $0.1 \mathrm{~m}$ for the six-channel independent PVSB system.

Fig. 4. Radiated sound power of the independent PVSB systems with different numbers of channels when the secondary loudspeaker plane is $0.1 \mathrm{~m}$ below the opening and the weighting factors are zero.

Fig. 5. Radiated sound power of the six-channel independent PVSB systems with different secondary loudspeaker locations when the weighting factors are zero.

Fig. 6. Convergence curves of the PVSB systems at $100 \mathrm{~Hz}$ with weighting factors of zero (a) two-channel systems (b) six-channel systems (c) fifteen-channel systems.

Fig. 7. Upper bound of the step size to ensure convergence for the six-channel systems with weighting factors of zero.

Fig. 8. Convergence curves of the six-channel PVSB systems with the maximum step size and weighting factors of zero.

Fig. 9. Experimental setup (a) the system overview, and (b) configuration of the PVSB system.

Fig. 10. Comparison between the simulated and measured sound power reduction with the secondary loudspeaker plane $0.1 \mathrm{~m}$ below the opening.

Fig. 11. Measured sound power reduction for (a) two-channel and six-channel systems with the secondary loudspeakers $0.1 \mathrm{~m}$ below the opening (b) six-channel systems with the secondary loudspeaker plane at different heights.

Fig. 12. Measured upper bound of the step size to ensure system convergence when the secondary loudspeaker plane is located with different intervals to the opening (a) two-channel systems with a $0.1 \mathrm{~m}$ interval (b) six-channel systems with a $0.1 \mathrm{~m}$ interval (c) six-channel systems with a $0.05 \mathrm{~m}$ interval (d) six-channel systems with a $0.01 \mathrm{~m}$ interval. 